\title{
Subclinical impairment of dynamic left ventricular systolic and diastolic function in patients with obstructive sleep apnea and preserved left ventricular ejection fraction
}

Antonello D'Andrea ${ }^{1 *+}$, Angelo Canora ${ }^{2 \dagger}$, Simona Sperlongano ${ }^{3}$, Domenico Galati ${ }^{4}$, Serena Zanotta ${ }^{4}$, Giorgio Emanuele Polistina ${ }^{2}$, Carmine Nicoletta², Giacomo Ghinassi ${ }^{2}$, Maurizio Galderisi $i^{\wedge}$, Alessandro Sanduzzi Zamparelli ${ }^{2}$, Patrizio Lancellotti ${ }^{6}$ and Marialuisa Bocchino ${ }^{2^{*}}$ (I)

\begin{abstract}
Background: Hypoxia affects myocardial oxygen supply resulting in subclinical cardiac dysfunction in obstructive sleep apnea (OSA) patients, with cardiovascular complications being associated with increased oxidative burst (OB). The aims of our study were to assess left ventricular (LV) dynamic myocardial deformation and diastolic reserve at rest and upon exercise, along with $\mathrm{OB}$ determination in this patients subset.

Methods: Conventional echocardiography, Doppler myocardial imaging and LV 2D speckle tracking echocardiography were performed in 55 OSA patients with preserved LV ejection fraction (EF) and 35 age and sex-comparable healthy controls. Peripheral OB levels were evaluated by flow cytometry.

Results: Despite comparable LVEF, LV global longitudinal strain (GLS) was significantly reduced in OSA at rest $(-13.4 \pm 3.8$ vs $-18.4 \pm 3.3$ in controls, $P<0.001)$ and at peak exercise $(-15.8 \pm 2.6$ vs $-23.4 \pm 4.3, P<0.001)$. Systolic pulmonary artery pressure (sPAP) and E/E' ratios increase during effort were higher in OSA than in controls ( $\triangle$ sPAP $44.3 \% \pm 6.4 \mathrm{vs} 32.3 \% \pm 5.5$, $P<0.0001$, and $\Delta E / E^{\prime} 87.5 \% \pm 3.5$ vs $25.4 \% \pm 3.3, P<0.0001$, respectively). The best correlate of $E / E^{\prime}$ at peak stress was peak exertion capacity $(r=-0.50, P<0.001)$. OB was also increased in OSA patients $(P=0.001)$ but, unlike OSA severity, was not associated with LV diastolic dysfunction.
\end{abstract}

Conclusions: Evaluation of diastolic function and myocardial deformation during exercise is feasible through stress echocardiography. OSA patients with preserved LVEF show subclinical LV systolic dysfunction, impaired LV systolic and diastolic reserve, reduced exercise tolerance, and increased peripheral levels of OB. Therapy aimed at increasing LV diastolic function reserve might improve the quality of life and exercise tolerability in OSA patients.

Keywords: Obstructive sleep apnea, Exercise echocardiography, 2D speckle tracking echocardiography, Diastolic function, Oxidative burst

\footnotetext{
*Correspondence: antonellodandrea@libero.it; marialuisa.bocchino@unina.it

${ }^{\dagger}$ Antonello D'Andrea and Angelo Canora contributed equally to this work.

"This study is dedicated to the memory of Prof. Maurizio Galderisi, prematurely passed away because of COVID-19.

${ }^{1}$ Unit of Cardiology and Intensive Care, Umberto I Hospital, Viale San

Francesco, 84014 Nocera Inferiore (Salerno), Italy

${ }^{2}$ Department of Clinical Medicine and Surgery, Respiratory Medicine Section, Federico II University (at Monaldi Hospital), Via L. Bianchi, 5, 80131 Naples, Italy

Full list of author information is available at the end of the article
}

(c) The Author(s). 2020 Open Access This article is licensed under a Creative Commons Attribution 4.0 International License, which permits use, sharing, adaptation, distribution and reproduction in any medium or format, as long as you give appropriate credit to the original author(s) and the source, provide a link to the Creative Commons licence, and indicate if changes were made. The images or other third party material in this article are included in the article's Creative Commons licence, unless indicated otherwise in a credit line to the material. If material is not included in the article's Creative Commons licence and your intended use is not permitted by statutory regulation or exceeds the permitted use, you will need to obtain permission directly from the copyright holder. To view a copy of this licence, visit http://creativecommons.org/licenses/by/4.0/ The Creative Commons Public Domain Dedication waiver (http://creativecommons.org/publicdomain/zero/1.0/) applies to the data made available in this article, unless otherwise stated in a credit line to the data. 


\section{Background}

Obstructive sleep apnea (OSA) is characterized by repeated episodes of partial or complete upper airways collapse, resulting in apnea and hypopnea events, intermittent hypoxemia and frequent arousals [1]. OSA is an increasing health problem, often associated with cardiovascular disorders, including left (LV) and right ventricular (RV) dysfunction, arterial hypertension, coronary artery disease, heart failure, and arrhythmias [2, 3]. Repetitive hypoxia episodes adversely affect the interaction between myocardial oxygen demand and supply, with development of subclinical systolic dysfunction [4]. Furthermore, hormonal and metabolic dysregulation, oxidative burst $(\mathrm{OB})$, systemic inflammation and mechanical hemodynamic disturbances lead to LV remodeling and diastolic dysfunction [5-8]. There is evidence of a consistent relationship between $\mathrm{OB}$ and OSA [9]. OB has been suggested as a marker of upper airway obstructive episodes and hypoxemia causing local oropharyngeal inflammation [10], and has been associated with cardiovascular complications [11, 12].

On this basis, we hypothesized that OSA patients with preserved LV ejection fraction (LVEF) would have dynamic abnormalities in LV myocardial deformation and/or increased dynamic diastolic stiffness. The aims of our study were to assess, by means of 2D speckle tracking echocardiography (2DSTE) and diastolic parameters, LV myocardial deformation and diastolic function indexes both at rest and during exercise, mainly focusing on the systolic and diastolic reserve. Reproducibility of 2DSTE measurements was also assessed. In addition, we evaluated peripheral levels of $\mathrm{OB}$ as we aimed to address any relationship with basal and dynamic heart function parameters.

\section{Methods}

\section{Study population}

From October 2017 to May 2018, 55 consecutive patients affected by moderate-severe OSA, referred to our Respiratory Medicine Division, were enrolled. Patients with a clinical history of concomitant lung disease (including chronic obstructive pulmonary disease, bronchial asthma, interstitial lung diseases), coronary artery disease, valvular heart disease, congestive heart failure, arrhythmias, and pulmonary hypertension were excluded. All OSA patients were not being treated with continuous positive airway pressure (CPAP). Thirty-five age- and sex-comparable healthy subjects referred to our attention for a voluntary cardiovascular screening were enrolled as controls. The study was conducted in accordance with the amended Declaration of Helsinki. The local Ethics committee approved the study and all individuals gave written informed consent.

\section{OSA assessment}

OSA diagnosis was performed with home overnight cardiorespiratory polygraphy using a VitalNight data acquisition and analysis system (AirLiquide Medical System, Rangendigen, Germany) according to reference guidelines [13]. Data were analysed by trained sleep physicians with more than 3-yr experience and OSA severity was graded with the apnea/hypopnea index (AHI) according to accepted criteria [13]. Nocturnal respiratory failure (NRF) was defined if $t 90 \%$ was $\geq 30 \%$ or there was at least one period of $5 \mathrm{~min}$ minimum with a $\mathrm{SpO}_{2} \leq$ $90 \%$ with a nadir of $85 \%$ during registration $[14,15]$. t90 is the percentage of time spent with a $\mathrm{SpO}_{2}<90 \%$.

\section{Lung function}

Spirometry, lung volumes measurement and determination of the haemoglobin $(\mathrm{Hb})$-adjusted single-breath diffusing lung capacity of the carbon monoxide $\left(\mathrm{DLCO}_{\mathrm{sb}}\right)$ were performed using a computer-assisted spirometer (Quark PFT 2008 Suite Version Cosmed Ltd., Rome, Italy) according to international standards [16-18]. The $6 \mathrm{~min}$ walking test $(6 \mathrm{MWT})$ was performed by trained hospital staff according to guidelines [19]. Arterial blood gas analysis at rest was also obtained.

\section{Heart study}

Standard trans-thoracic echocardiography, Doppler evaluation and strain analysis were performed at rest and at peak effort using market available equipment (Vivid E9 GE Healthcare, Milwaukee, WI, USA; MyLab Alpha ESAOTE, Florence, Italy). All measurements were performed at our Cardiology Unit and independently assessed by two cardiologists expert in echocardiography, according to current recommendations [20-22]. Rest echocardiography: LV ejection fraction (EF) and LV global longitudinal strain (GLS) were evaluated as systolic function indexes. LVEF was calculated using the Simpson biplane method, from the apical 4- and 2-chamber views. A biplane LVEF $\geq 52 \%$ for men and $\geq 54 \%$ for women were considered normal [20]. For strain calculation, the endocardial borders of the LV myocardial walls were traced by a point-and-click approach, in the end-systolic frame of the 2D images, from the apical 3-, 4-, and 2-chamber views. The tracking algorithm followed the endocardium during all the cardiac cycle. Basal, mid, and apical regions of interest were created and segments that failed to track were manually adjusted. Longitudinal strains for each of 18 segments were measured and LV GLS was calculated as the mean strain of all the segments. The tracking process and conversion to Lagrangian strains were performed offline using dedicated software (EchoPAC PC 2D strain, GE Healthcare, Milwaukee, WI, USA). We defined impaired GLS as $>-20 \%$ (a less negative value reflects a more impaired GLS) [20, 21]. The following diastolic 
function parameters were measured by pulsed wave (PW) Doppler and tissue Doppler imaging (TDI), in apical 4chamber view: peak early (E) and late (A) diastolic velocity of the mitral inflow, E/A ratio, peak septal and lateral early myocardial diastolic velocity (e') and average E/e' ratio. The peak tricuspid regurgitation velocity (TRV) was measured in multiple echocardiographic windows. On the basis of the highest TRV obtained, systolic pulmonary artery pressure (sPAP) was calculated through the Bernoulli's principle: $\left(4 \mathrm{XTRV}^{2}\right)+$ right atrial pressure (RAP). RAP was estimated by measuring the diameter of the inferior vena cava and its respiratory motion. Left atrial volume index (LAVI) was assessed through biplane area-length method, dividing the left atrial volume by the body surface area (BSA). Atrium acquisitions were made from the apical 4- and 2 - chamber views. The presence of more than 2 between average $E / e^{\prime}>14$, septal $e^{\prime}<7 \mathrm{~cm} / \mathrm{s}$ or lateral $\mathrm{e}^{\prime}<10 \mathrm{~cm} / \mathrm{s}, \quad$ TRV $>2.8 \mathrm{~m} / \mathrm{s} \quad(\mathrm{sPAP}>36 \mathrm{mmHg}), \quad$ and LAVI $>34 \mathrm{ml} / \mathrm{m}^{2}$, was considered expression of LV diastolic dysfunction in subjects with normal LVEF [22]. Exercise stress echocardiography: Standard supine bicycle exercise stress echocardiography was performed with incremental steps of $25 \mathrm{~W}$ every 2 min [23]. Parameters evaluated at peak exercise included LV GLS as systolic function index, LV diastolic parameters (E, septal and lateral e' and average E/e' ratio) and sPAP. An increase in the E/e' ratio and/or sPAP upon exercise were considered expression of impaired LV diastolic function reserve [23].

\section{Oxidative burst determination}

Oxidative burst of peripheral leukocytes was measured with the Phagoburst BURSTEST ${ }^{\mathrm{mix}}$ (PHAGOBURST $^{\mathrm{mix}}$, BD Bioscences, La Jolla, CA, USA), according to the manufacturer's instruction. Briefly, $100 \mu \mathrm{l}$ of heparinized whole blood was incubated with opsonized E.coli at $37^{\circ} \mathrm{C}$ for $10 \mathrm{~min}$. A sample without stimulus served as negative background control. Dihydrorhodamine (DHR) 123 was added for $10 \mathrm{~min}$ to allow the conversion to fluorescent rhodamine 123 upon reactive oxygen species (ROS) production. After erythrocytes were removed and washing, $200 \mu \mathrm{l}$ of DNA staining solution was added for $10 \mathrm{~min}$ to exclude aggregation artifacts. Samples were acquired with a FACS CANTO flow cytometer (BD Biosciences, La Jolla, CA, USA). Analysis was performed with the FACS DIVA software.

\section{Statistical analysis}

Statistical analyses were performed using a commercially available package (SPSS, Rel. 21.0. 2016, SPSS Inc., Chicago, IL, USA). Variables are presented as mean \pm standard deviation (SD). Two-tailed $t$-test for paired and unpaired data was used to assess changes between groups.

Linear regression analyses and partial correlation test by Pearson's method were used to assess univariate relations. The following variables were included into the analysis: clinical parameters (age, heart rate, oxygen

Table 1 Demographics and clinical features, lung function parameters, and sleep-related findings of the study population

\begin{tabular}{llll}
\hline Variable & $\begin{array}{l}\text { OSA } \\
(n=55)\end{array}$ & $\begin{array}{l}\text { Controls } \\
(n=35)\end{array}$ & P-value \\
\hline $\begin{array}{l}\text { Age (years) } \\
\text { Gender, male sex }\end{array}$ & $39.9 \pm 8.8$ & $50.2 \pm 5.4$ & NS \\
Smoking habit & & $24(68)$ & NS \\
$\quad$ Smokers & $9(16)$ & $0(0)$ & \\
Former smokers & $23(42)$ & $0(0)$ & \\
No smokers & $23(42)$ & $35(100)$ & \\
Pack/yr & $32.8 \pm 24.6$ & & \\
BMI (Kg/m $\left.{ }^{2}\right)$ & $32.9 \pm 6.7$ & $28.3 \pm 4.7$ & $<\mathbf{0 . 0 1}$ \\
Dyslipidemia (\%) & $10(18)$ & $0(0)$ & \\
Hypertension (\%) & $14(25)$ & $0(0)$ & \\
Diabetes (\%) & $12(22)$ & $0(0)$ &
\end{tabular}

Lung function parameters

$\begin{array}{llll}\text { FVC (\%pred.) } & 85.4 \pm 13.6 & 98 \pm 12.5 & <0.001 \\ \mathrm{FEV}_{1} \text { (\%pred.) } & 87 \pm 16.8 & 86 \pm 13 & \mathrm{NS} \\ \mathrm{FEV}_{1} / \mathrm{FVC} \text { (\%) } & 102.7 \pm 15.4 & 84 \pm 9.5 & <\mathbf{0 . 0 0 1} \\ \mathrm{TLC} \text { (\% pred) } & 85 \pm 5.9 & 86 \pm 3.8 & \mathrm{NS} \\ \mathrm{DLCO}_{\mathrm{sb}} \text { (\% pred) } & 84.3 \pm 16.1 & 86 \pm 5 & \mathrm{NS} \\ \mathrm{pH} & 7.38 \pm 0.3 & 7.39 \pm 0.4 & \mathrm{NS} \\ \mathrm{PaO}_{2} \text { (mmHg) } & 84.7 \pm 8.1 & 85.3 \pm 6.2 & \mathrm{NS} \\ \mathrm{PaCO}_{2} \text { (mmHg) } & 39.8 \pm 4.1 & 38.6 \pm 4.5 & \mathrm{NS} \\ \text { Lactates (mmol/L) }^{-} \text {(m) } & 1.2 \pm 1.4 & 1.1 \pm 1.2 & \mathrm{NS} \\ \mathrm{HCO}^{-} \text {(mmol/L) } & 26.1 \pm 1.6 & 25.2 \pm 1.4 & \mathrm{NS} \\ \text { 6MWT mt } & 512.1 \pm 175.1 & 568 \pm 145.6 & \mathrm{NS} \\ \text { Sleep-related events } & & & \\ \text { TST (minutes) } & 358.4 \pm 68.7 & 362 \pm 64.5 & \mathrm{NS} \\ \text { Supine time (\%) } & 49.7 \pm 33.8 & 41.2 \pm 29.8 & \mathrm{NS} \\ \text { AHI } & 46.3 \pm 28.7 & 3.2 \pm 1.2 & <0.0001 \\ \text { Supine AHI (events/hour) } & 41.4 \pm 27 & 2.2 \pm 2.4 & <0.0001 \\ \text { ODI } & 46 \pm 30.7 & 3.3 \pm 1.2 & <0.0001 \\ \text { t90 (\%) } & 24 \pm 22.2 & 0.4 \pm 0.6 & 0.001 \\ \text { Nadir \% SpO } & 68.5 \pm 11.5 & 83.2 \pm 6.2 & 0.0001\end{array}$

Data are expressed as absolute number (\%) or mean \pm SD. Statistically significant results $(p<0.05)$ are reported in bold

Abbreviations: BMI Body mass index, FVC Forced vital capacity, $F E V_{1}$ Forced expiratory volume in the 1 st second, $T L C$ Total lung capacity, $D L C O_{s b}$ Single breath carbon monoxide lung diffusing capacity, $\mathrm{PaO}_{2}$ Oxygen arterial partial pressure, $\mathrm{PaCO}_{2}$ Carbon dioxide arterial partial pressure, $\mathrm{HCO} 3$ Sodium bicarbonates, $6 M W T \mathrm{mt}$, Meters traveled during 6 min walking test, TST Total sleep time, AHI Apnea/hypopnea index, ODI Oxygen desaturation index, $t 90 \%$ Percentage of time spent with $\mathrm{SpO}_{2}<90 \%, \mathrm{SpO}_{2}$ Arterial oxygen saturation 
saturation, systolic blood pressure, diastolic blood pressure, body mass index (BMI), risk factors, comorbidities); lung function, sleep-related and metabolic parameters; standard echocardiographic and 2DSTE parameters. To identify significant independent determinants of resting and dynamic LV diastolic function in OSA patients, their individual association with clinical, functional and echocardiographic variables was assessed by multivariate analysis, using a bidirectional stepwise regression. Variables included in our analysis were all the variables significantly $(p<0.05)$ associated with the explained variable by univariate analysis. Odds ratios (OR) were calculated using a logistic regression method, and Beta-coefficients were obtained by linear regression method. Values of $p<0.05$ were considered significant for all analyses.

Receiver operating characteristic (ROC) curve analysis was performed to select optimal cut-off values of echocardiographic parameters. Reproducibility of GLS measurements was determined in all the subjects. Intra-observer variability and inter-observer variability were examined using the coefficient of variation (COV), defined as the ratio of the standard deviation $(\sigma)$ to the mean $(\mu)(\%)$, and by Bland-Altman analysis. COV, 95\% confidence intervals (CIs), and percent errors were reported.

\section{Results}

\section{Peripheral levels of oxidative burst are increased in OSA} patients

Our study population was composed of 90 subjects, including 55 OSA patients and 35 healthy controls. Main demographic and clinical characteristics are summarized in Table 1. Lung function was preserved in both study groups (Table 1), with the exception of forced vital capacity that was slightly lower in OSA patients. Distribution of sleep-related respiratory events is reported in
Table 1 . Thirty patients were suffering from severe OSA (54\%), while $21(39 \%)$ had a concomitant condition of NRF, as assessed by a $t 90>30 \%$ in all cases. In line with previous observations [9-11], levels of OB were significantly increased in OSA patients than in controls (Fig. 1). OB was significantly higher in patients with a $190>30 \%$, with no differences according to the AHI.

\section{LV systolic function is impaired at rest and at peak exercise in moderate-severe OSA with a good reproducibility of 2DSTE measurements}

LVEF was similar in OSA and control cohorts, and preserved in both (Table 2). LV mass was mildly increased in OSA with no significant difference in LV diameters in the 2 groups (Table 2). LV speckle tracking was obtainable at rest in $95 \%$ of the total analysed segments. The remaining $5 \%$ segments were not considered due to a suboptimal tracking score. In OSA patients, LV GLS was significantly reduced at rest, this variation being statistically meaningful in all segments analysed (Table 2, Fig. 2). Also LV GLS increase upon exercise was lower as compared to controls (Table 3).

Overall, we observed a good reproducibility of global LV strain measurements in our study population, similar to that reported for speckle-tracking-based strain measures performed in selected samples - including healthy young adults $[20,21]$. The average coefficients of variation were $\leq 7 \%$ for the longitudinal strain measures. For all analyzed measures, intra- and intra-observer ICC values were $\geq 0.80$ (Intra-observer variability: COV: LV GLS: 5.37 (ICC 0.73); BlandAltman analysis: LV GLS (95\% CI \pm 1.5 ; percent error 3.2\%). Inter-observer variability: COV: LV GLS: 7.22 (ICC 0.77); Bland-Altman analysis: LV GLS (95\% CI \pm 1.7 ; percent error $3.6 \%$ ).

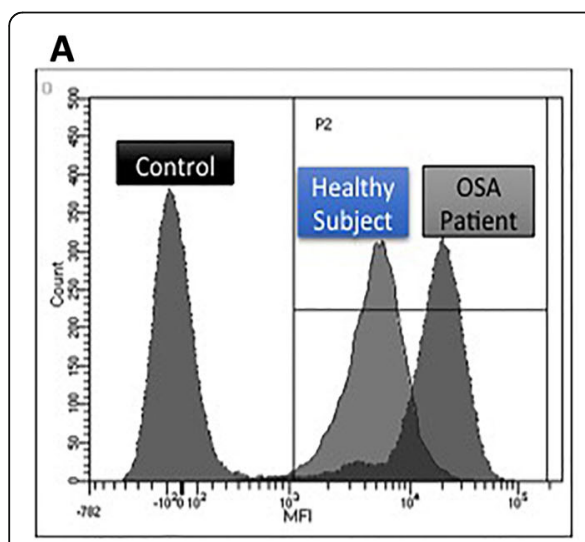

B

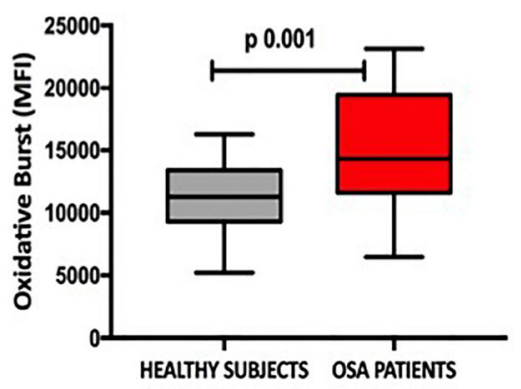

C

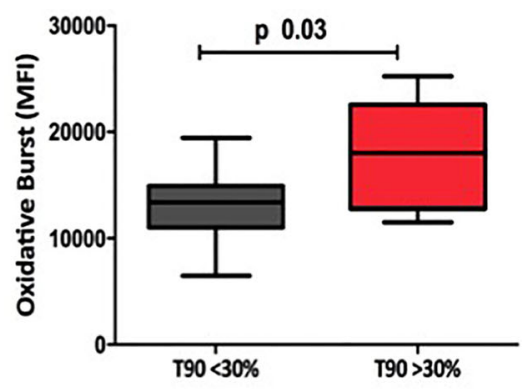

Fig. 1 Levels of oxidative burst in OSA patients and healthy controls. a Distribution of oxidative burst (OB), calculated as mean fluorescence intensity (MFI), in OSA patients with respect to healthy volunteers; b Boxplot showing that peripheral levels of OB (MFI) are significantly increased in OSA patients as compared to healthy volunteers; c Boxplot showing the distribution of OB (MFI) in OSA patients according to t90\%. As reported, OB levels are significantly increased in patients with t90 > 30\% 
Table 2 Left ventricle standard echo and 2D speckle tracking echo measurements at rest and peak effort

\begin{tabular}{|c|c|c|c|}
\hline Variable & $\begin{array}{l}\text { OSA } \\
(n=55)\end{array}$ & $\begin{array}{l}\text { Controls } \\
(n=35)\end{array}$ & $P$-value \\
\hline \multicolumn{4}{|l|}{ Resting measurements } \\
\hline $\mathrm{SpO}_{2}(\%)$ & $94.7 \pm 16.2$ & $98 \pm 1.6$ & $<0.01$ \\
\hline IVSd (mm) & $11.4 \pm 1.8$ & $9.1 \pm 2.3$ & $<0.01$ \\
\hline PWd (mm) & $10.4 \pm 1.6$ & $8.7 \pm 2.1$ & $<0.01$ \\
\hline LVEDD (mm) & $48.3 \pm 3.9$ & $47.2 \pm 4.4$ & NS \\
\hline LVESD (mm) & $34.3 \pm 3.6$ & $32.4 \pm 4.1$ & NS \\
\hline LV mass index $\left(\mathrm{g} / \mathrm{m}^{2}\right)$ & $52.8 \pm 5.3$ & $48.1 \pm 3.4$ & $<0.01$ \\
\hline Biplane LVEF (\%) & $56.5 \pm 6.2$ & $57.4 \pm 5.5$ & NS \\
\hline LV GLS (\%) & $-13.4 \pm 3.8$ & $-18.4 \pm 3.3$ & $<0.001$ \\
\hline Mitral E velocity $(\mathrm{cm} / \mathrm{s})$ & $0.9 \pm 0.3$ & $0.8 \pm 0.4$ & NS \\
\hline Mitral A velocity $(\mathrm{cm} / \mathrm{s})$ & $0.7 \pm 0.4$ & $0.9 \pm 0.3$ & NS \\
\hline E/A ratio & $1.2 \pm 0.4$ & $0.9 \pm 0.4$ & $<0.01$ \\
\hline Mitral septal e' velocity $(\mathrm{cm} / \mathrm{s})$ & $0.13 \pm 0.02$ & $0.16 \pm 0.05$ & $<0.01$ \\
\hline Mitrallateral e' velocity $(\mathrm{cm} / \mathrm{s})$ & $0.14 \pm 0.03$ & $0.17 \pm 0.03$ & $<0.01$ \\
\hline E/e' ratio & $8.2 \pm 3.1$ & $5.9 \pm 2.8$ & $<0.01$ \\
\hline LAVI $\left(\mathrm{ml} / \mathrm{m}^{2}\right)$ & $32.4 \pm 4.4$ & $28.3 \pm 5.1$ & $<0.01$ \\
\hline $\mathrm{sPAP}(\mathrm{mmHg})$ & $31.5 \pm 7.8$ & $21.3 \pm 2.9$ & $<0.01$ \\
\hline TAPSE (mm) & $22.5 \pm 3.3$ & $24.5 \pm 3.8$ & NS \\
\hline Tricuspid $\mathrm{S}^{\prime}$ velocity $(\mathrm{cm} / \mathrm{s})$ & $13.3 \pm 2.2$ & $14.4 \pm 3.1$ & NS \\
\hline \multicolumn{4}{|l|}{ Peak effort measurements } \\
\hline $\mathrm{SpO}_{2}(\%)$ & $92.3 \pm 3.2$ & $97.2 \pm 2.6$ & $<0.01$ \\
\hline Exercise capacity (Watt) & $115.3 \pm 25$ & $150.4 \pm 35$ & $<0.001$ \\
\hline Biplane LVEF (\%) & $62.3 \pm 5.8$ & $65.7 \pm 6.8$ & NS \\
\hline LV GLS (\%) & $-15.8 \pm 2.6$ & $-23.4 \pm 4.3$ & $<0.001$ \\
\hline Mitral E velocity $(\mathrm{cm} / \mathrm{s})$ & $1.1 \pm 0.5$ & $1.2 \pm 0.6$ & NS \\
\hline Mitral A velocity $(\mathrm{cm} / \mathrm{s})$ & $0.9 \pm 0.4$ & $0.9 \pm 0.3$ & NS \\
\hline E/A ratio & $1.2 \pm 0.4$ & $1.3 \pm 0.5$ & $<0.01$ \\
\hline Mitral septal e' velocity $(\mathrm{cm} / \mathrm{s})$ & $0.08 \pm 0.02$ & $0.18 \pm 0.05$ & $<0.0001$ \\
\hline Mitral lateral e' velocity $(\mathrm{cm} / \mathrm{s})$ & $0.07 \pm 0.03$ & $0.19 \pm 0.03$ & $<0.0001$ \\
\hline E/e' ratio & $15.4 \pm 4.1$ & $7.4 \pm 2.8$ & $<0.0001$ \\
\hline $\mathrm{sPAP}(\mathrm{mmHg})$ & $45.5 \pm 5.8$ & $28.4 \pm 4.2$ & $<0.001$ \\
\hline TAPSE (mm) & $26.4 \pm 2.3$ & $27.5 \pm 5.8$ & NS \\
\hline Tricuspid s' velocity (cm/s) & $16.4 \pm 3.1$ & $17.7 \pm 4.1$ & NS \\
\hline
\end{tabular}

Data are expressed as mean \pm SD. Statistically significant results $(p<0.05)$ are reported in bold

Abbreviations: $\mathrm{SpO}_{2}$ Arterial oxygen saturation, IVSd Inter-ventricular septum thickness at end diastole, PWd Posterior wall thickness at end diastole, LVEDD Left ventricular end diastolic diameter, LVESD Left ventricular end systolic diameter, $L V$ Left ventricle, $L V E F$ Left ventricular ejection fraction, $L V$ GLS Left ventricular global longitudinal strain, $L A V I$ Left atrial volume index, SPAP Systolic pulmonary artery pressure, TAPSE Tricuspid annular plane systolic excursion

\section{LV diastolic function is impaired at rest and at peak} exercise in moderate-severe OSA

E/e' ratio, sPAP and LAVI at rest were all significantly higher in patients affected by OSA as compared to healthy controls; moreover, both septal and lateral e' velocities were significantly lower in the former (Table 2, Fig. 2). According to recent guidelines, a definite diastolic dysfunction was present at rest in 19 OSA (34.5\%) [22].

$\mathrm{E} / \mathrm{e}^{\prime}$ increase during exertion was significantly higher in patients affected by OSA when compared to controls; sPAP increase (difference between peak exertion and resting sPAP) also showed the same trend along with a three-fold decrease of oxygen saturation (Table 3).

\section{Exercise tolerance is reduced in moderate-severe OSA}

During physical exercise, OSA patients showed a reduced tolerance with a lower maximal workload in Watts $(115.3 \pm 25$ vs $150.4 \pm 35, p<0.001)$ and oxygen saturation level $(92.3 \% \pm 3.2$ vs $97.2 \% \pm 2.6, p<0.01)$ as compared to healthy controls (Table 2).

\section{Resting and exercise-induced LV diastolic dysfunction is associated with OSA severity but not with OB levels}

Logistic regression analysis showed that arterial hypertension (OR 2.67, 95\% CI 1.74-5.61; $p<0.01$ ), resting LV GLS > - 15\% (OR 3.3, 95\% CI 2.34-4.15; $p<0.001$ ) and severe AHI (OR 2.96, 95\% CI 1.92-4.91; $p<0.001$ ) were independently associated with LV diastolic dysfunction at rest.

At peak exercise, by linear regression analysis E/e' ratio was directly related to peak exertion capacity expressed in Watts (beta coefficient $-0.50, p<0.001$ ), to blood lactates at rest (beta coefficient $0.36, p<0.01$ ), to resting LV GLS (beta coefficient 0.46, $p<0.001$ ), and to the AHI (beta coefficient 0.42, $p<0.001$ ) (Table 4). No significant correlations were found with $\mathrm{OB}, \mathrm{BMI}$ smoking, and co-morbidities.

\section{Discussion}

OSA is frequently associated with adverse clinical outcomes [1], including cardiovascular diseases [24-27]. In the present study, we showed that OSA patients: (1) have subclinical LV systolic dysfunction despite a preserved LV output, and a low contractile reserve as compared to healthy controls; (2) have an impaired LV diastolic reserve along with a reduced exercise tolerance; (3) display higher levels of $\mathrm{OB}$ that are not correlated with diastolic heart function.

In 2007, Gondi et al. reported that OSA was associated with sleep-induced LV systolic longitudinal dysfunction, as measured by pulsed wave Doppler and tissue Doppler imaging (TDI) [28]. As TDI is technically limited by the insonation angle of the ultrasound beam, we further proved by 2DSTE, which is independent of the interrogation angle, that LV and RV early myocardial dysfunction occur in OSA patients and are associated with disease severity [29]. The relationship between OSA and LV systolic dysfunction is not surprising as OSA patients 


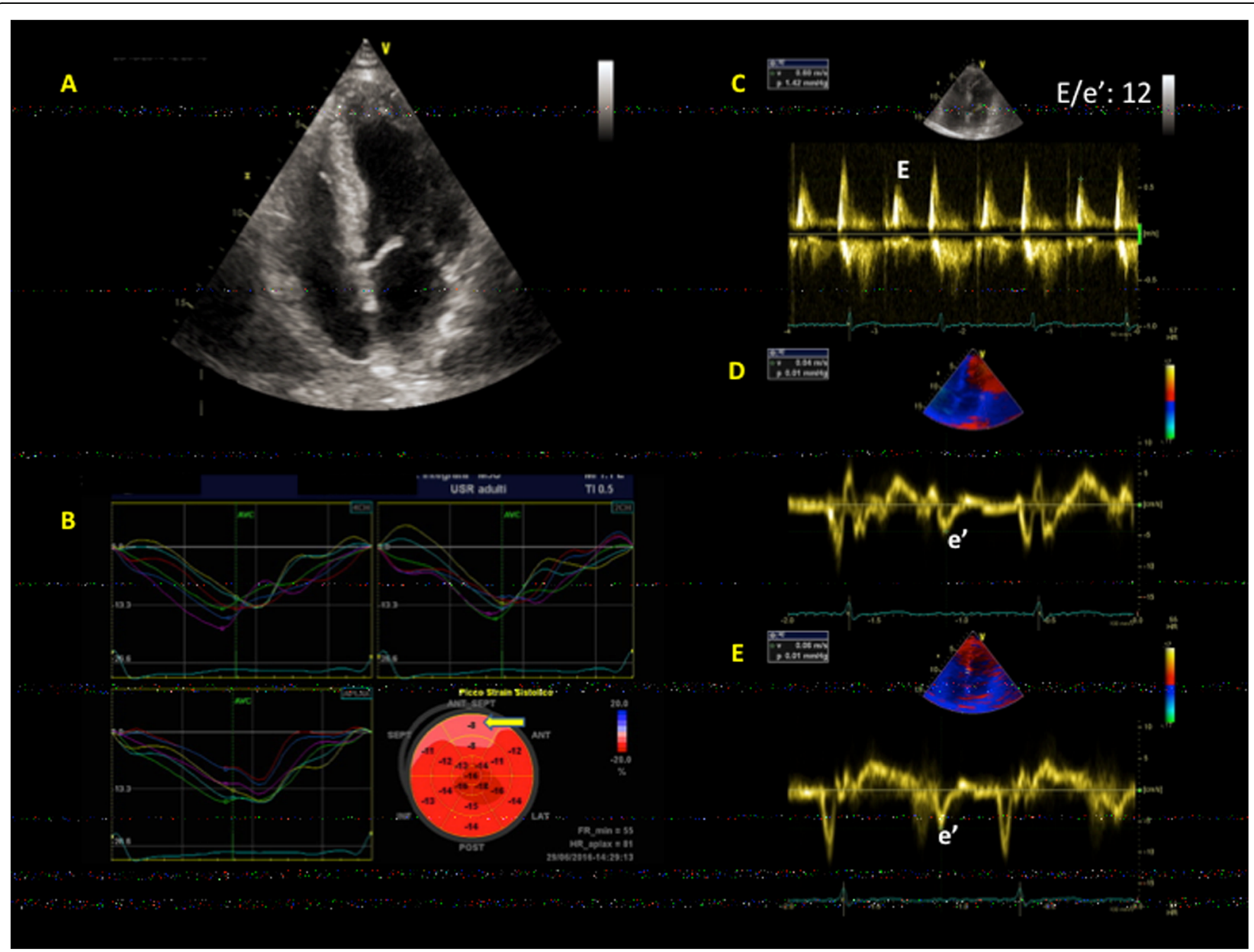

Fig. 2 Left ventricular systolic and diastolic dysfunction in OSA patients. Two-dimensional echocardiography (a: apical four chamber view) showing mild impairment of resting LV regional and global strain (mainly in the septal region, see arrow) (b) and significant diastolic dysfunction assessed by transmitral flow pattern (c) and both lateral (d) and septal (e) pulsed Doppler tissue imaging

experience repeated increases in LV after-load during sleep, due to exaggerated negative intra-thoracic pressure and intermittent hypoxia and arousals. Acute repeated increases in after-load can therefore result in LV subclinical systolic dysfunction [29]. In this study, we enrolled patients with moderate-severe OSA and healthy controls with preserved LVEF and found an early

Table 3 Changes in echocardiographic parameters and oxygen saturation in OSA and controls during effort

\begin{tabular}{llll}
\hline Variable & $\begin{array}{l}\text { OSA } \\
(n=55)\end{array}$ & $\begin{array}{l}\text { Controls } \\
(n=35)\end{array}$ & -value \\
\hline$\Delta$ sPAP (\%) & $44.3 \pm 6.4$ & $32.3 \pm 5.5$ & $<\mathbf{0 . 0 0 1}$ \\
$\Delta$ E/e' ratio (\%) & $87.5 \pm 3.5$ & $25.4 \pm 3.3$ & $<\mathbf{0 . 0 0 0 1}$ \\
$\Delta \mathrm{LV} \mathrm{GLS} \mathrm{( \% )}$ & $15.8 \pm 3.4$ & $25.4 \pm 4.1$ & $<\mathbf{0 . 0 0 1}$ \\
$\Delta \mathrm{SpO}_{2}(\%)$ & $-2.5 \pm 3.3$ & $-0.8 \pm 2.8$ & $<\mathbf{0 . 0 1}$
\end{tabular}

Data are expressed as mean \pm SD. Statistically significant results $(p<0.05)$ are reported in bold

Abbreviations: SPAP Systolic pulmonary artery pressure, LV GLS Left ventricular global longitudinal strain, $\mathrm{SpO}_{2}$ Arterial oxygen saturation involvement of the LV myocardium in the former. Actually, despite LVEF, that is traditionally used as systolic function parameter, was normal in OSA patients, we found an impaired basal LV GLS, which is expression of subclinical LV pump dysfunction. In addition, we also found that strain increase during physical exercise in OSA patients was lower if compared to healthy controls, reflecting their low contractile reserve. Interestingly, we previously detected that OSA patients have also a

Table 4 Multivariate analysis model: correlation between E/e' ratio at peak exercise with univariable clinical parameters

\begin{tabular}{llll}
\hline Variable & & Beta coefficient & $P$-value \\
\hline LV E/e' at peak & Watts (at peak effort) & -0.50 & $<\mathbf{0 . 0 0 1}$ \\
exercise & Blood lactates (at rest) & 0.36 & $<\mathbf{0 . 0 1}$ \\
& LV GLS (at rest) & 0.46 & $<0.001$ \\
& AHI & 0.42 & $<\mathbf{0 . 0 0 1}$ \\
\hline
\end{tabular}

Statistically significant results $(p<0.05)$ are reported in bold

Abbreviations: $L V$ GLS Left ventricular global longitudinal strain, $A H I$ Apnea-hypopnea index 
subclinical RV dysfunction, and that RV GLS impairment was associated with SPAP and disease severity [30].

Previous studies show that the prevalence of LV diastolic dysfunction at rest among OSA patients varies from 23 to $56 \%$, depending on the sample size and the method of diastolic function assessment [31-34]. In our cohort we observed that, according to recent guidelines [22], a definite diastolic dysfunction was present in the $34.5 \%$ of OSA patients at rest. Pathophysiological mechanisms underlying LV diastolic dysfunction in OSA have been widely studied: repetitive hypoxemia/re-oxygenation sequences, sympathetic bursts, renin-angiotensinaldosterone system activation, oxidative stress, systemic inflammation, intra-thoracic pressure reduction and trans-mural pressure increasing, all participate in the development of LV hypertrophy and remodelling, and hence of LV diastolic dysfunction [5-8].

To our knowledge this is the first study addressing the LV diastolic performance both at rest and at peak exercise in a selected population of OSA patients. In middle-aged healthy subjects, the $\mathrm{E} / \mathrm{e}^{\prime}$ ratio does not change significantly with exercise because of proportional increases in both the mitral inflow and annular velocities [35-38]. Conversely, in our series almost all patients enrolled showed an increase of the E/e' ratio, that was significantly higher than in the control group. This finding indicates an impairment of the LV diastolic function reserve resulting in an increased LV filling pressure during exercise [22, 39, 40]. In agreement with these observations, also SPAP increase was significantly higher in OSA than in healthy subjects. The mean sPAP value in OSA patients was $>43 \mathrm{mmHg}$ (as estimated by a TRV $>3.1 \mathrm{~m} / \mathrm{s}$ ), which is the echocardiography threshold of abnormal diastolic stress according to international guidelines [23]. The present study clearly underlines the strong relation between $\mathrm{E} / \mathrm{e}^{\prime}$ ratio and exercise tolerance in OSA. Patients with a higher E/e' ratio at peak exercise, and hence a more impaired diastolic reserve, showed a reduced functional capacity highlighted by a lower maximal workload and a reduced peak $\mathrm{SpO}_{2}$ level. The E/e' ratio at peak exercise also resulted significantly correlated to LV GLS, to AHI, which reflects OSA severity, and to blood lactates, which are suggestive of inadequate tissue perfusion. These findings can be explained by the mentioned pathological mechanisms responsible for myocardial remodelling and fibrosis. The latter provokes impairment in both systolic and diastolic function, which can worsen during exercise, leading to reduced functional capacity.

Our study has some limitations. First, it is a single-centre study with a sample size too small to drive definitive general conclusions concerning subclinical LV functional impairment in OSA patients. Also, our findings cannot necessarily be extrapolated to patients with mild disease. Finally, despite we found no correlations between the diastolic impairment during exercise and patients age, $\mathrm{OB}$, BMI, smoking and co-morbidities, we cannot definitely exclude their contribution. Indeed, while we excluded OSA patients with major heart and lung diseases, the prevalence of cardiovascular risk factors, such as smoke, obesity, dyslipidemia, diabetes, and hypertension, was still not negligible in our patient cohort. Further studies in larger populations will certainly help to address their impact by ad hoc sub-group analyses.

\section{Conclusions}

In conclusion, evaluation of diastolic function and myocardial deformation during exercise is feasible through stress echocardiography and provides valuable information in predicting exercise capacity in a selected population of moderate-severe OSA patients with preserved LVEF. These patients show subclinical LV systolic dysfunction, impaired LV systolic and diastolic reserve, reduced exercise tolerance, and increased peripheral levels of OB. A CPAP treatment and a therapy aimed at increasing LV diastolic function reserve might improve the quality of life and exercise tolerability in this target population.

\section{Abbreviations \\ 2DSTE: 2D speckle tracking echocardiography; 6-MWT: 6-min walk test; A: Late diastolic velocity of the mitral inflow; AHI: Apnea/hypopnea index; BSA: Body surface area; CPAP: Continuous positive airway pressure (CPAP); DHR: Dihydrorhodamine; $\mathrm{DLCO}_{\mathrm{sb}}$ : Single-breath diffusing lung capacity of the carbon monoxide; E: Early diastolic velocity of the mitral inflow; e': Early myocardial diastolic velocity; EF: Ejection fraction; GLS: Global longitudinal strain; LAVI: Left atrial volume index; LV: Left ventricular; NRF: Nocturnal respiratory failure; OB: Oxidative burst; OSA: Obstructive sleep apnea; PW: Pulsed wave; RAP: Right atrial pressure; ROC: Receiver operating characteristic; ROS: Reactive oxygen species; RV: Right ventricular; SPAP: Systolic pulmonary artery pressure; $\mathrm{SpO}_{2}$ : Oxygen saturation; TDI: Tissue Doppler imaging; TRV: Tricuspid regurgitation velocity}

\section{Acknowledgments}

The present work has been presented as abstract at the 80th SIC National Italian Congress 2019 (European heart Journal Supplements 2019; 21: Supplement J- The Heart of the matter; J73-N1).

\section{Authors' contributions}

$\mathrm{ADA}$ and $\mathrm{AC}$ equally contributed. $\mathrm{ADA}, \mathrm{AC}$ and $\mathrm{MB}$ : conception and design of the study, data acquisition and analysis, and manuscript writing. SS MG and PL: echocardiography data acquisition and analysis. DG and SZ: flow cytometry data acquisition and analysis. GEP, CN, GG, AS: patients recruitment. All Authors approved the final version of the manuscript.

\section{Funding}

No funding.

\section{Availability of data and materials}

The datasets used and/or analysed during the current study are available from the corresponding author on reasonable request.

\section{Ethics approval and consent to participate}

The study was conducted in accordance with the amended Declaration of Helsinki. The "Federico II" Istitutional Ethical Committee, Naples, Italy approved the study (Protocol 1129, 4 August 2015) and all individuals gave written informed consent. 


\section{Consent for publication}

Not applicable

\section{Competing interests}

Prof. M. Bocchino and Prof A. Sanduzzi are associate editors of BMC Pulm Med. The remaining Authors have no conflicting interests for this study.

\begin{abstract}
Author details
${ }^{1}$ Unit of Cardiology and Intensive Care, Umberto I Hospital, Viale San Francesco, 84014 Nocera Inferiore (Salerno), Italy. ${ }^{2}$ Department of Clinical Medicine and Surgery, Respiratory Medicine Section, Federico II University (at Monaldi Hospital), Via L. Bianchi, 5, 80131 Naples, Italy. ${ }^{3}$ Unit of Cardiology, Department of Translational Medical Sciences, University of Campania "Luigi Vanvitelli", Monaldi Hospital, Naples, Italy. ${ }^{4}$ Haematology-Oncology and Stem Cell Transplantation Unit, Department of Haematology and Innovative Therapies, Istituto Nazionale Tumori- IRCCS Fondazione G. Pascale, Naples, Italy. ${ }^{5}$ Department of Advanced Biomedical Sciences, Federico II University Hospital Via S. Pansini 5, Naples, Italy. ${ }^{6} \mathrm{CHU}$ de Liége, Service de Cardiologie, Liege, Belgium.
\end{abstract}

\section{Received: 8 August 2019 Accepted: 24 February 2020}

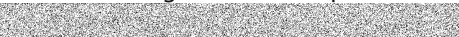

\section{References}

1. Qaseem A, Dallas P, Owens DK, Starkey M, Holty JEC, Shekelle P. Diagnosis of obstructive sleep apnea in adults: a clinical practice guideline from the American College of Physicians. Ann Intern Med. 2014;161(3):210-20.

2. Phillips B. Sleep-disordered breathing and cardiovascular disease. Sleep Med Rev. 2005:9(2):131-40.

3. Leung RST, Bradley TD. State of the art sleep apnea and cardiovascular disease. Am J Respir Crit Care Med. 2001;164(12):2147-65.

4. Haruki N, Takeuchi M, Kanazawa Y, et al. Continuous positive airway pressure ameliorates sleep-induced subclinical left ventricular systolic dysfunction: demonstration by two-dimensional speckle-tracking echocardiography. Eur J Echocardiogr. 2010;11(4):352-8.

5. Bodez D, Damy T, Soulat-Dufour L, Meuleman C, Cohen A. Consequences of obstructive sleep apnoea syndrome on left ventricular geometry and diastolic function. Arch Cardiovasc Dis. 2016;109(8-9):494-503.

6. Shim CY, Kim D, Park S, et al. Effects of continuous positive airway pressure therapy on left ventricular diastolic function: a randomised, sham-controlled clinical trial. Eur Respir J. 2018:51:1701774.

7. Cetin S, Vural M, Akdemir R, Firat H. Left atrial remodelling may predict exercise capacity in obstructive sleep apnoea patients. Acta Cardiol. 2018; 73(5):471-8

8. Butt M, Dwivedi G, Shantsila A, Khair OA, Lip GYH. Left ventricular systolic and diastolic function in obstructive sleep apnea impact of continuous positive airway pressure therapy. Circ Heart Fail. 2012:5(2):226-3.

9. Lira $A B$, de Sousa Rodrigues CF. Evaluation of oxidative stress markers in obstructive sleep apnea syndrome and additional antioxidant therapy: a review article. Sleep Breath. 2016;20:1155-60.

10. Passali D, Corallo G, Yaremchuk S, et al. Oxidative stress in patients with obstructive sleep apnoea syndrome. Acta Otorhinolaryngol Ital. 2015;35(6): 420-5.

11. Hopps E, Lo Presti R, Montana M, Canino B, Calandrino V, Caimi G. Analysis of the correlations between oxidative stress, gelatinases and their tissue inhibitors in the human subjects with obstructive sleep apnea syndrome. J Physiol Pharmacol. 2015;66(6):803-10.

12. Asker S, Asker M, Sarikaya E, Sunnetcioglu A, Aslan M, Demir H. Oxidative stress parameters and their correlation with clinical, metabolic and polysomnographic parameters in severe obstructive sleep apnea syndrome. Int J Clin Exp Med. 2015;8(7):11449-55 eCollection 2015.

13. Berry RB, Budhiraja R, Gottlieb DJ, et al. Rules for scoring respiratory events in sleep: update of the 2007 AASM manual for the scoring of sleep and associated events. J Clin Sleep Med. 2012;8(5):597-619.

14. Levi-Valensi P, Weitzenblum E, Rida Z, et al. Sleep-related oxygen desaturation and daytime pulmonary haemodynamics in COPD patients. Eur Respir J. 1992:5(3):301-7.

15. Fletcher EC, Miller J, Divine GW, Fletcher JG, Miller T. Nocturnal oxyhemoglobin desaturation in COPD patients with arterial oxygen tensions above $60 \mathrm{~mm}$ hg. Chest. 1987;92(4):604-8.
16. Miller MR, Hankinson J, Brusasco V, et al. Standardisation of spirometry. Eur Respir J. 2005;26(2):319-38.

17. Wanger J, Clausen $J \mathrm{~L}$, Coates A, et al. Standardisation of the measurement of lung volumes. Eur Respir J. 2005;26(3):511-22.

18. Maclntyre N, Crapo RO, Viegi G, et al. Standardisation of the single-breath determination of carbon monoxide uptake in the lung. Eur Respir J. 2005: 26(4):720-35.

19. ATS Committee on Proficiency Standards for Clinical Pulmonary Function Laboratories. ATS statement: guidelines for the six-minute walk test. Am J Respir Crit Care Med. 2002;166(1):111-7.

20. Lang RM, Badano LP, Mor-Avi V, et al. Recommendations for cardiac chamber quantification by echocardiography in adults: an update from the American Society of Echocardiography and the European Association of Cardiovascular Imaging. Eur Heart J Cardiovasc Imaging. 2015;16(3):233-70.

21. Voigt JU, Pedrizzetti G, Lysyansky P, et al. Definitions for a common standard for 2D speckle tracking echocardiography: consensus document of the EACVI/ASE/Industry Task Force to standardize deformation imaging. Eur Heart J Cardiovasc Imaging. 2015;16(1):1-11.

22. Nagueh SF, Smiseth OA, Appleton CP, et al. Recommendations for the evaluation of left ventricular diastolic function by echocardiography: an update from the American Society of Echocardiography and the European Association of Cardiovascular Imaging. J Am Soc Echocardiogr. 2016;29(4): 277-314.

23. Lancellotti P, Pellikka PA, Budts W, et al. The clinical use of stress echocardiography in non-Ischaemic heart disease: recommendations from the European Association of Cardiovascular Imaging and the American Society of Echocardiography. Eur Heart J Cardiovasc Imaging. 2016 Novi 17(11):1191-229.

24. Marin JM, Carrizo SJ, Vicente E, Agusti AG. Long-term cardiovascular outcomes in men with obstructive sleep apnoea-hypopnoea with or without treatment with continuous positive airway pressure: an observational study. Lancet. 2005;365(9464):1046-53.

25. Punjabi NM. The epidemiology of adult obstructive sleep apnea. Proc Am Thorac Soc. 2008:5(2):136-43.

26. Shahar E, Whitney CW, Redline S, et al. Sleep-disordered breathing and cardiovascular disease: cross-sectional results of the sleep heart health study. Am J Respir Crit Care Med. 2001;163(1):19-25.

27. Yaggi HK, Concato J, Kernan WN, Lichtman JH, Brass LM, Mohsenin V. Obstructive sleep apnea as a risk factor for stroke and death. N Engl J Med. 2005;353(19):2034-41.

28. Gondi S, Dokainish H. Right ventricular tissue Doppler and strain imaging: ready for clinical use? Echocardiography. 2007;24(5):522-32.

29. D'Andrea A, Martone F, Liccardo B, et al. Acute and chronic effects of noninvasive ventilation on left and right myocardial function in patients with obstructive sleep apnea syndrome: a speckle tracking echocardiographic study. Echocariography. 2016;33(8):1144-55.

30. Buonauro A, Galderisi M, Santoro C, et al. Obstructive sleep apnoea and right ventricular function: a combined assessment by speckle tracking and three-dimensional echocardiography. Int J Cardiol. 2017;243:544-9.

31. Bodez D, Lang S, Meuleman C, et al. Left ventricular diastolic dysfunction in obstructive sleep apnoea syndrome by an echocardiographic standardized approach: an observational study. Arch Cardiovasc Dis. 2015;108(10):480-90.

32. Arias MA, Garcia-Rio F, Alonso-Fernandez A, Mediano O, Martinez I, Villamor J. Obstructive sleep apnea syndrome affects left ventricular diastolic function: effects of nasal continuous positive airway pressure in men. Circulation. 2005;112(3):375-83.

33. Baguet JP, Barone-Rochette $G$, Levy $P$, et al. Left ventricular diastolic dysfunction is linked to severity of obstructive sleep apnoea. Eur Respir J. 2010;36(6):1323-9.

34. Fung JW, Li TS, Choy DK, et al. Severe obstructive sleep apnea is associated with left ventricular diastolic dysfunction. Chest. 2002;121(2):422-9.

35. Ha JW, Lulic F, Bailey KR, et al. Effects of treadmill exercise on mitral inflow and annular velocities in healthy adults. Am J Cardiol. 2003;91(1):114-5.

36. Oh JK, Kane GC. Diastolic stress echocardiography: the time has come for its integration into clinical practice. J Am Soc Echocardiogr. 2014; 27(10):1060-3.

37. Kane GC, Sachdev A, Villarraga HR, et al. Impact of age on pulmonary artery systolic pressures at rest and with exercise. Echo Res Prac. 2016;3(2):53-61.

38. Ha JW, Choi D, Park S, et al. Determinants of exercise-induced pulmonary hypertension in patients with normal left ventricular ejection fraction. Heart. 2009;95(6):490-4. 
39. Burgess MI, Jenkins C, Sharman JE, Marwick TH. Diastolic stress echocardiography: hemodynamic validation and clinical significance of estimation of ventricular filling pressure with exercise. J Am Coll Cardiol. 2006:47(9):1891-900.

40. Ha JW, Oh JK, Pellikka PA, et al. Diastolic stress echocardiography: a novel noninvasive diagnostic test for diastolic dysfunction using supine bicycle exercise Doppler echocardiography. J Am Soc Echocardiogr. 2005;18(1):63-8.

\section{Publisher's Note}

Springer Nature remains neutral with regard to jurisdictional claims in published maps and institutional affiliations.

Ready to submit your research? Choose BMC and benefit from:

- fast, convenient online submission

- thorough peer review by experienced researchers in your field

- rapid publication on acceptance

- support for research data, including large and complex data types

- gold Open Access which fosters wider collaboration and increased citations

- maximum visibility for your research: over $100 \mathrm{M}$ website views per year

At BMC, research is always in progress.

Learn more biomedcentral.com/submissions 